\title{
PRODUÇÃO DE BIOLUBRIFICANTES POR REAÇÃO DE HIDROESTERIFICAÇÃO CATALISADA POR LIPASES VEGETAL E MICROBIANA
}

\author{
A. P. P. BRESSANI ${ }^{1}$, K. C. A. GARCIA ${ }^{1}$, M. C. C. CUCATTI ${ }^{2}$, F. A. P. LAGE ${ }^{2}$, J. J. BASSI ${ }^{2}$, D. B. \\ HIRATA $^{2}$ e A. A. MENDES ${ }^{2}$ \\ ${ }^{1}$ Universidade Federal de São João del Rei, Departamento de Engenharia de Alimentos \\ ${ }^{2}$ Universidade Federal de Alfenas, Instituto de Química \\ E-mail para contato: adriano.mendes@unifal-mg.edu.br
}

\begin{abstract}
RESUMO - O presente estudo reporta a produção de biolubrificantes por hidroesterificação a partir do óleo da polpa de macaúba. Os ésteres foram obtidos por sequencial hidrólise mediada por extrato enzimático de sementes dormentes de mamona (produção de ácidos graxos livres - AGL), seguido da esterificação dos AGL purificados com diferentes álcoois em meio de heptano catalisada por lipase de Thermomyces lanuginosus (LTL) imobilizada por adsorção física em partículas de poli-hidróxibutirato (PHB). Hidrólise total do óleo de macaúba, otimizada por delineamento experimental completo "estrela rotacional", foi alcançada na razão mássica óleo:tampão de $35 \% \mathrm{~m} / \mathrm{m}$, $35^{\circ} \mathrm{C}$ e $6 \% \mathrm{~m} / \mathrm{m}$ de biocatalisador após $110 \mathrm{~min}$ de reação. Diferentes álcoois foram testados na esterificação de AGL purificados (razão equimolar álcool:AGL) e máxima conversão em éster $(\approx 92 \%)$ foi obtida para a reação com hexanol $(750 \mathrm{mM}$ de cada reagente) após 15 min de reação. O biocatalisador foi altamente ativo após oito bateladas consecutivas de esterificação.
\end{abstract}

\section{INTRODUÇÃO}

O mercado internacional de lubrificantes foi de 40,55 milhões de toneladas em 2012 e a projeção é de que o crescimento anual seja de 2,3\%, com estimativa de 43,9 milhões de toneladas em 2017. No Brasil, o consumo de lubrificantes cresceu 33\% durante o período de 2004 a 2012, atingindo 1,23 milhão de toneladas em 2012 (IHS, 2014). Estes compostos têm sido amplamente empregados como lubrificantes automotivos e fluidos hidráulicos. Entretanto, cerca de $50 \%$ destes lubrificantes são descartados no meio ambiente por meio de vazamentos, descartes inadequados, emulsões óleoágua, gases de exaustão de componentes, etc (Akerman et al., 2011). Alguns são resistentes à biodegradação, representando uma ameaça ao meio ambiente. Além disso, o aumento dos preços no mercado internacional e a crescente conscientização ecológica da sociedade têm conduzido à procura de alternativas sustentáveis aos lubrificantes minerais (Chowdhury et al., 2013; 2014).

Os triacilglicerois (óleos vegetais, gorduras animais e óleos residuais) têm-se mostrado uma alternativa bastante promissora na substitução de óleos minerais convencionais para a produção de lubrificantes, pois são estruturalmente semelhantes aos hidrocarbonetos de origem fóssil e com 


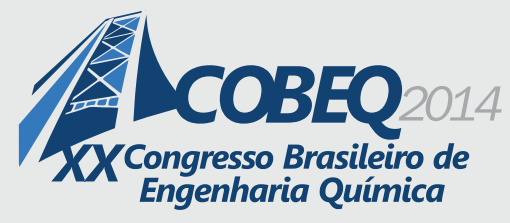

19 a 22 de outubro de 2014
Florianópolis/SC

características de serem renováveis, não-tóxicos e ecologicamente corretos (Chowdhury et al., 2013; 2014). Nos últimos anos, tem havido uma crescente demanda por lubrificantes ambientalmente "corretos" a partir de triacilglicerois de diferentes fontes, principalmente óleos vegetais. Apesar dos óleos vegetais serem muito similares aos lubrificantes minerais, estes compostos apresentam algumas desvantagens como elevada sensibilidade à hidrólise e à oxidação em elevadas temperaturas e solidificação em baixas temperaturas e, consequentemente, a sua aplicação se torna limitada (Akerman et al., 2011). Um dos fatores responsáveis pela instabilidade química dos óleos vegetais é a elevada reatividade da molécula de glicerol existente na estrutura química dos triacilglicerois (Gryglewicz et al., 2003).

A modificação química por reações de esterificação e transesterificação tem sido amplamente empregada com o intuito de superar estas limitações (Akerman et al., 2011; Chowdhury et al., 2013; 2014). Estas modificações permitem a produção de lubrificantes com excelentes propriedades físicoquímicas e elevada biodegradabilidade em condições aeróbias e anaeróbias (Akerman et al., 2011). Dentre os diferentes processos de modificação de triacilglicerois, a hidroesterificação, um processo que consite de hidrólise de triacilglicerois na produção de concentrados de AGL, seguida de esterificação com diferentes álcoois, tem mostrado ser uma estratégia na produção de biolubrificantes a partir de óleos com elevada concentração de ácidos graxos livres (Chowdhury et al., 2013; 2014).

Neste contexto, o objetivo deste projeto consiste na produção de biolubrificantes a partir de óleo não refinado da polpa de macaúba por hidroesterificação empregando o extrato enzimático bruto de sementes dormentes de mamona como biocatalisador na etapa de hidrólise, seguido da esterificação de AGL purificados com diferentes álcoois (álcool isoamílico, 2-etil-hexanol e hexanol) mediada por biocatalisador preparado a partir da imobilização de LTL em partículas de PHB.

\section{MATERIAIS E MÉTODOS}

\subsection{Materiais}

Sementes dormentes de mamona foram adquiridos da BRSeed Produção e Comércio de Sementes (Araçatuba/SP). Lipase de Thermomyces lanuginosus foi adquirida comercialmente da Sigma-Aldrich Co. (St. Louis, MO, EUA). Partículas hidrofóbicas de poli-hidróxibutirato (PHB) (área superficial de $17 \mathrm{~m}^{2} / \mathrm{g}$ e diâmetro de poro de $3,1 \mathrm{~nm}$ ) foram adquiridos da PHB Industrial (São Paulo/SP). Óleo de macaúba com acidez de 7,7 mg KOH/g de óleo foi adquirido da Paradigma Óleos Vegetais Ltda. (Carmo do Parnaíba/MG). Álcoois isoamílico, 2-etil-hexanol e 1-hexanol foram adquiridos da Sigma-Aldrich Co. Todos os outros reagentes empregados foram de grau analítico adquiridos das empresas Vetec Química e Synth (São Paulo/SP).

\subsection{Preparação de biocatalisadores}

Extrato enzimático bruto de sementes dormentes de mamona: A preparação do extrato enzimático foi realizada conforme metodologia descrita por Santos et al. (2013). As sementes foram descascadas, trituradas em moinho de facas e incubadas em acetona a frio para a extração do óleo e o extrato bruto obtido foi empregado como biocatalisador nas reações de hidrólise do óleo de macaúba. 


\section{9 a 22 de outubro de 2014 \\ Florianópolis/SC}

Imobilização de LTL em PHB por adsorção física: A imobilização da lipase em PHB foi realizada em tampão fosfato de sódio $\mathrm{pH}$ 7,0 $(5 \mathrm{mM})$ por oferecendo carregamento de proteína de 40 $\mathrm{mg} / \mathrm{g}$ de suporte (Miranda et al., 2014). A suspensão suporte:solução enzimática (relação 1:20) foi mantida por $12 \mathrm{~h} \mathrm{a} 25^{\circ} \mathrm{C}$ sob agitação em shaker $(200 \mathrm{rpm})$. Ao final, o biocatalisador preparado foi filtrado e lavado com água destilada. O biocatalisador preparado exibiu atividade hidrolítica (hidrólise do azeite de oliva emulsificado) e concentração de proteína imobilizada de $1240 \pm 30 \mathrm{UI} / \mathrm{g}$ e 24,7 \pm $1,1 \mathrm{mg} / \mathrm{g}$ de suporte, respectivamente (Miranda et al., 2014).

\subsection{Otimização da hidrólise enzimática do óleo de macaúba por delineamento experimental}

$\mathrm{Na}$ hidrólise do óleo de macaúba mediada por extrato enzimático bruto de sementes dormentes de mamona, foi proposto um delineamento experimental completo "estrela rotacional" para melhor entendimento da influência individual das variáveis estudadas e de suas interações na reação de hidrólise. Os parâmetros avaliados foram a razão mássica óleo:tampão, temperatura de reação e concentração de biocatalisador. Como variável resposta foi considerada a porcentagem de hidrólise, determinada de acordo com Santos et al. (2013). Os resultados obtidos foram analisados empregando o software Statistica versão 7.0. As reações de hidrólise foram conduzidas em frascos contendo $50 \mathrm{~g}$ de meio (óleo + tampão acetato $\mathrm{pH} \mathrm{4,5-100} \mathrm{mM)} \mathrm{sob} \mathrm{vigorosa} \mathrm{agitação} \mathrm{mecânica} \mathrm{(1000} \mathrm{rpm)} \mathrm{por} 30$ min de incubação. Após este estudo, foram realizados ensaios adicionais de hidrólise do óleo de macaúba variando a velocidade de agitação (100-1500 rpm) e concentração de íons cálcio (0-10 mM). Em condições otimizadas, a hidrólise do óleo de macaúba foi conduzida por um período máximo de 120 min para verificar o tempo mínimo requerido para obter máxima porcentagem de hidrólise.

\subsection{Purificação dos ácidos graxos livres}

Um excesso de hexano (relação 1:2 v/v) foi adicionado à mistura reacional. A mistura foi então centrifugada a $5000 \mathrm{rpm}$ e $25{ }^{\circ} \mathrm{C}$ por $20 \mathrm{~min}$. A fase superior, que consistiu de uma mistura de hexano e AGL, foi separada da fase aquosa (glicerol, biocatalisador e solução tampão), na parte inferior. A fase orgânica foi filtrada a vácuo e o solvente foi removido por evaporador rotativo. Finalmente, sulfato de sódio anidro foi adicionado aos AGL previamente purificados para a remoção de traços de moléculas de água.

\subsection{Síntese enzimática de ésteres (biolubrificantes) por esterificação em meio orgânico}

A reação de esterificação dos AGL purificados com diferentes álcoois (álcool isoamílico, hexanol e 2-etil-hexanol) foi conduzida em meio de heptano, empregando razão molar AGL:álcool (1:1), de acordo com metodologia previamente descrita por Miranda et al. (2014). A solução de substrato $(10 \mathrm{~mL})$ foi incubada com 1,6 $\mathrm{g}$ de biocatalisador preparado por imobilização de LTL em PHB. As reações foram conduzidas a $32,5{ }^{\circ} \mathrm{C}$ por um período máximo de 30 min sob agitação contínua em shaker $(200 \mathrm{rpm})$. A porcentagem de conversão em éster foi estimada por determinação da concentração de AGL residual por titulação com solução de $\mathrm{NaOH}(20 \mathrm{mM})$, utilizando 


\section{9 a 22 de outubro de 2014 \\ Florianópolis/SC}

fenolftaleína como indicador (Miranda et al., 2014). Todos os experimentos foram realizados em triplicata. Reações controle foram também realizados por incubação de partículas de PHB nas mesmas condições de ensaio. Sob estas condições, não foi observado consumo de AGL após 30 min de incubação. Testes de estabilidade operacional ( 8 bateladas consecutivas de 15 min cada) também foram conduzidos para o sistema reacional com máxima atividade de esterificação nas mesmas condições reacionais acima descritas. Ao final de cada batelada, o biocatalisador foi removido por filtração a vácuo e lavado com heptano a frio para a remoção de substratos não convertidos e produtos da reação adsorvidos no microambiente do biocatalisador e incubado em uma nova solução de susbtrato.

\section{RESULTADOS E DISCUSSÃO}

\subsection{Otimização da produção de AGL por hidrólise enzimática}

A produção de AGL a partir da hidrólise enzimática do óleo de macaúba foi mediada por extrato enzimático bruto de sementes dormentes de mamona, um biocatalisador de baixo custo e com elevada atividade hidrolítica. A escolha do biocatalisador foi baseada em estudos anteriores visando a produção de AGL a partir de diferentes óleos vegetais (Santos et al., 2013). As reações foram realizadas em tampão acetato de sódio pH 4,5 (100 mM). Sob estas condições, o biocatalisador exibe máxima atividade hidrolítica (Santos et al., 2013).

No presente estudo, foi proposto um planejamento fatorial completo "estrela rotacional" para verificar os parâmetros que influenciam significativamente a hidrólise enzimática do óleo de macaúba. A Tabela 1 mostra as variáveis independentes e os níveis codificados e originais, bem como os valores experimentais e preditos de porcentagem de hidrólise. Os resultados sumarizados na Tabela 1 indicam que a percentagem de hidrólise variou entre 11,5 (ensaios 12 e 13) e 40\% (ensaio 10) após 30 min de reação. Estes resultados também revelam boa correspondência entre os valores experimentais e preditos, o que implica que o modelo empírico proposto pode ser empregado para descrever adequadamente a relação entre as variáveis e a resposta (percentagem de hidrólise).

Os resultados da Tabela 1 foram utilizados para estimar os efeitos principais variáveis e as suas interações. A análise estatística mostrou efeitos significativos para os termos lineares de razão mássica óleo:tampão $\left(\mathrm{x}_{1}\right)$, temperatura de reação $\left(\mathrm{x}_{2}\right)$ e concentração de biocatalisador $\left(\mathrm{x}_{3}\right)$ e os termos quadráticos de temperatura de reação $\left(\mathrm{x}_{2}{ }^{2}\right)$ e concentração de biocatalisador $\left(\mathrm{x}_{3}{ }^{2}\right)$ a $95 \%$ de nível de confiança. Os coeficientes relativos aos efeitos não significativos foram excluídos do modelo. A equação 1 representa os dados experimentais obtidos na Tabela 1, cuja porcentagem de variação explicada $\left(\mathrm{R}^{2}\right)$ foi de $94,44 \%$.

$$
\text { Hidrólise }(\%)=37.16+2.86 x_{1}-3.10 x_{2}-6.67 \mathrm{x}_{2}{ }^{2}+4.59 x_{3}-4.97 x_{3}{ }^{2}
$$

Em que: $\mathrm{x}_{1}, \mathrm{x}_{2}$ e $\mathrm{x}_{3}$ representam os valores codificados para a razão mássica óleo:tampão, temperatura de reação e concentração de biocatalisador, respectivamente. 
Tabela 1 - Planejamento experimental para a hidrólise do óleo de macaúba catalisada por lipase de sementes dormentes de mamona em reator tanque-agitado

\begin{tabular}{lccccc}
\hline \multirow{2}{*}{ Ensaios } & \multicolumn{2}{c}{ Variáveis codificadas (originais) } & \multicolumn{2}{c}{ Hidrólise (\%) } \\
\cline { 2 - 6 } & $\begin{array}{c}\text { Razão mássica } \\
\text { óleo:tampão } \\
(\% \mathrm{~m} / \mathrm{m})\end{array}$ & $\begin{array}{c}\text { Temperatura de } \\
\text { reação }\end{array}$ & $\begin{array}{c}\text { Biocatalisador } \\
(\% \mathrm{~m} / \mathrm{m})\end{array}$ & $\begin{array}{c}\text { Valor } \\
\text { experimental }\end{array}$ & $\begin{array}{c}\text { Valor } \\
\text { predito }\end{array}$ \\
\hline 1 & $-1(20)$ & $-1(30)$ & $-1(3)$ & $21,4 \pm 1,3$ & 21,2 \\
2 & $+1(40)$ & $-1(30)$ & $-1(3)$ & $30,3 \pm 2,1$ & 26,9 \\
3 & $-1(20)$ & $+1(45)$ & $-1(3)$ & $17,5 \pm 0,6$ & 15,0 \\
4 & $+1(40)$ & $+1(45)$ & $-1(3)$ & $23,1 \pm 1,5$ & 20,7 \\
5 & $-1(20)$ & $-1(30)$ & $+1(7)$ & $33,8 \pm 3,5$ & 30,3 \\
6 & $+1(40)$ & $-1(30)$ & $+1(7)$ & $36,1 \pm 0,5$ & 36,1 \\
7 & $-1(20)$ & $+1(45)$ & $+1(7)$ & $21,9 \pm 1,3$ & 24,1 \\
8 & $+1(40)$ & $+1(45)$ & $+1(7)$ & $32,1 \pm 2,6$ & 29,9 \\
9 & $-1,68(13,2)$ & $0(37,5)$ & $0(5)$ & $32,9 \pm 1,5$ & 32,3 \\
10 & $+1,68(46,8)$ & $0(37,5)$ & $0(5)$ & $40,1 \pm 1,9$ & 42,0 \\
11 & $0(30)$ & $-1,68(24,9)$ & $0(5)$ & $20,7 \pm 1,1$ & 23,5 \\
12 & $0(30)$ & $+1,68(50,1)$ & $0(5)$ & $11,6 \pm 0,8$ & 13,1 \\
13 & $0(30)$ & $0(37,5)$ & $-1,68(1,64)$ & $11,7 \pm 1,1$ & 15,4 \\
14 & $0(30)$ & $0(37,5)$ & $+1,68(8,36)$ & $30,2 \pm 0,9$ & 30,8 \\
15 & $0(30)$ & $0(37,5)$ & $0(5)$ & $37,2 \pm 1,1$ & 37,2 \\
16 & $0(30)$ & $0(37,5)$ & $0(5)$ & $37,4 \pm 0,7$ & 37,2 \\
17 & $0(30)$ & $0(37,5)$ & $0(5)$ & $36,5 \pm 0,8$ & 37,2 \\
18 & $0(30)$ & $0(37,5)$ & $0(5)$ & $35,3 \pm 2,6$ & 37,2 \\
\hline
\end{tabular}

Máxima porcentagem de hidrólise do óleo de macaúba foi alcançada a $35{ }^{\circ} \mathrm{C}$ e nos níveis máximos de razão mássica óleo:tampão (46,8\% em massa de óleo) e concentração de biocatalisador de $6 \% \mathrm{~m} / \mathrm{m}$ após 30 min de reação sob vigorosa agitação $(1000 \mathrm{rpm})$, condições determinadas pela análise das superfícies de resposta geradas pelo modelo proposto. Para a validação do modelo proposto, foi realizada a hidrólise do óleo de macaúba em triplicata sob as condições experimentais descritas acima para verificar o modelo proposto, determinada pela aplicação da equação de regressão (Eq. 1). O valor predito pelo modelo foi de $42,1 \%$ e o experimental foi de $42,5 \pm 3,1 \%$ em 30 min de reação. Os resultados indicam que o modelo gerado permitiu uma previsão adequada da produção de AGL por hidrólise enzimática do óleo de polpa de macaúba.

Em seguida, foi verificada a influência da velocidade de agitação na porcentagem de hidrólise do óleo nas condições experimentais previamente otimizadas. De acordo com a Figura 1, o aumento da velocidade de agitação aumentou significativamente a porcentagem de hidrólise até $1000 \mathrm{rpm}$. Entretanto, acima de $1000 \mathrm{rpm}$ não foi observado um aumento na porcentagem de hidrólise. Com base nestes resultados, a velocidade de agitação de $1000 \mathrm{rpm}$ foi suficiente para permitir uma boa difusão de gotículas de óleo do meio reacional para o microambiente do biocatalisador (extrato enzimático bruto). 


\section{9 a 22 de outubro de 2014 \\ Florianópolis/SC}

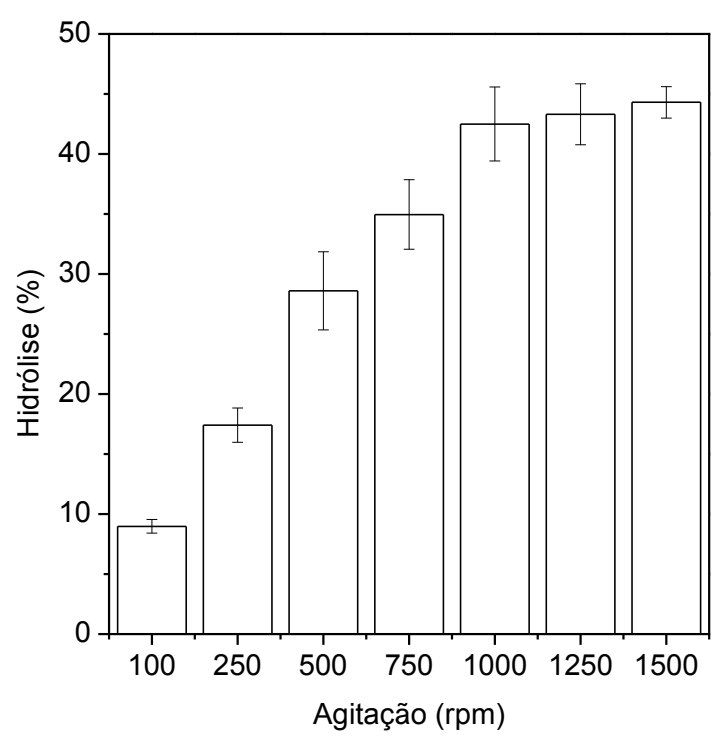

Figura 1 - Influência da velocidade de agitação na porcentagem de hidrólise do óleo de macaúba mediada pelo extrato enzimático bruto.

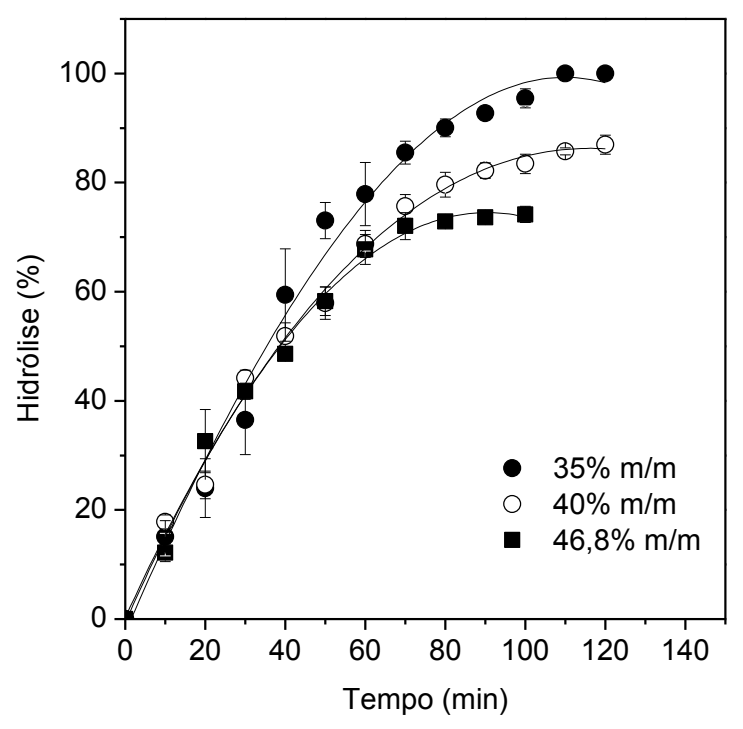

Figura 2 - Perfil cinético de hidrólise enzimática do óleo de macaúba em diferentes razões mássicas óleo:tampão.

Nas condições otimizadas, foi realizada a hidrólise enzimática do óleo de macaúba em diferentes razões mássicas óleo:tampão, como mostrada na Figura 2. As reações foram conduzidas por um período máximo de $2 \mathrm{~h}$ sob vigorosa agitação $(1000 \mathrm{rpm})$. Na razão mássica de 46,8\% m/m, previamente selecionada pelo delineamento experimental, máxima percentagem de hidrólise foi de $74,1 \pm 1,5 \%$ após 100 min de reação. Deste modo, reações com menor concentração de óleo na solução de substrato foram conduzidas. De acordo com a Figura 2, foi possível verificar que a $40 \%$ $\mathrm{m} / \mathrm{m}$ foi observada máxima percentagem de hidrólise de $87,0 \pm 1,8 \%$, após 120 min de reação. Entretanto, total hidrólise do óleo vegetal foi alcançada somente para a reação realizada na razão mássica óleo:tampão de $35 \% \mathrm{~m} / \mathrm{m}$. Esta redução na percentagem de hidrólise por aumento da concentração de óleo é creditada pelo aumento da concentração de AGL na interface óleo/água que promove possíveis alterações do estado de ionização da enzima, afetando, portanto, a sua atividade catalítica (Santos et al., 2013). Com base nestes resultados, a hidrólise enzimática do óleo de macaúba para a produção de concentrados de AGL pode ser eficientemente realizada na razão mássica de $35 \%$ nas condições experimentais anteriormente otimizadas pela metodologia de superfície de resposta.

\subsection{Produção de biolubrificantes por reação de esterificação mediada por lipase microbiana imobilizada em partículas de PHB}

Como mostrado acima, o extrato enzimático bruto de sementes dormentes de mamona apresentou alta atividade catalítica em reações de hidrólise de óleo vegetal. Entretanto, estudos preliminares realizados em nosso laboratório demonstraram a sua baixa atividade catalítica em reações em meio orgânico como transesterificação e esterificação (dados não mostrados). 
Consequentemente, um biocatalisador alternativo preparado pela imobilização de LTL por adsorção física em partículas hidrofóbicas de PHB foi testado na produção de ésteres alquílicos (biolubrificantes) por esterificação direta de AGL e diferentes álcoois em meio orgânico. Estudos mostram a sua elevada atividade catalítica em reações de esterificação (Miranda et al., 2014).

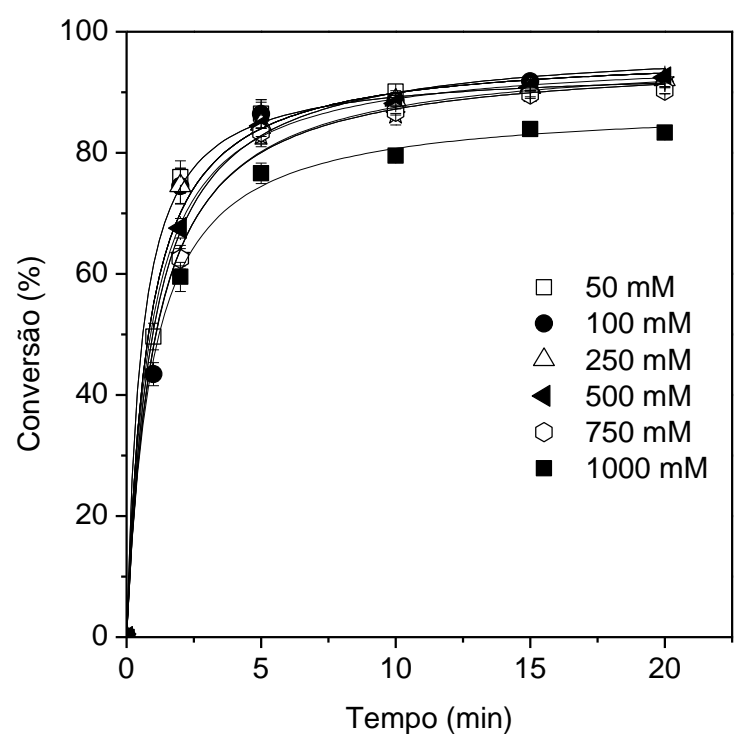

Figura 3 - Perfil cinético de produção de ésteres de hexila por esterificação em meio de heptano mediada por LTL imobilizada em PHB em diferentes concentrações de reagentes.

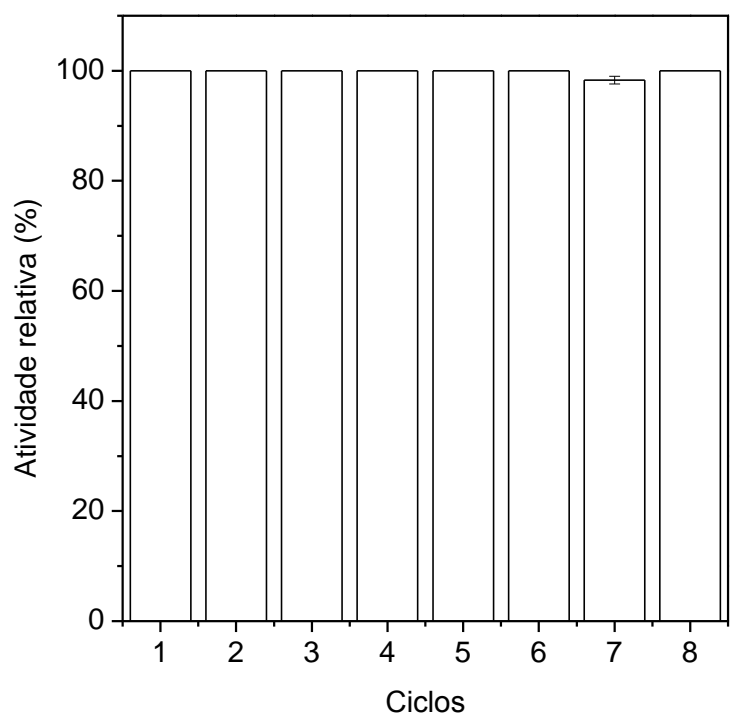

Figura 4 - Estabilidade operacional de LTL imobilizada em PHB em bateladas consecutivas de produção de ésteres de hexila em meio de heptano.

Neste estudo, ésteres alquílicos foram produzidos por esterificação de AGL com álcoois isoamílico, 2-etil-hexanol e 1-hexanol em diferentes concentrações. As reações foram realizadas em razão molar álcool:AGL $(1: 1)$ a $32,5^{\circ} \mathrm{C}$, sob agitação em shaker $(200 \mathrm{rpm})$ por 30 min de reação empregando $16 \% \mathrm{~m} / \mathrm{m}$ de biocatalisador (Miranda et al., 2014). A aplicação de álcool isoamílico e 2etil-hexanol resultou em máxima porcentagem de esterificação da ordem de 85 e 70\%, respectivamente (dados não mostrados). Entretanto, máxima conversão em ésteres $(92,4 \pm 0,4 \%)$ foi alcançada após 15 min de reação empregando 1-hexanol como aceptor acila (Figura 3). As reações realizadas com concentrações de reagentes $\leq 750 \mathrm{mM}$ exibiu similar conversão. Entretanto, a reação conduzida a $1000 \mathrm{mM}$ resultou em uma ligeira redução da porcentagem de esterificação devido ao aumento da acidez no sistema reacional que reduz a atividade catalítica do biocatalisador (Miranda et $a l ., 2014)$. Testes de estabilidade operacional também foram realizados em consecutivos ciclos de esterificação de AGL e 1-hexanol a $750 \mathrm{mM}$ de cada reagente em meio de heptano. A Figura 4 mostra que o biocatalisador preparado não apresentou perda de sua atividade catalítica após oito sucessivos ciclos de esterificação. De acordo com estes resultados, é possível observar que o biocatalisador testado (LTL imobilizada em PHB) é um biocatalisador altamente promissor na produção de compostos de interesse industrial como ésteres de hexila (biolubrificantes). 


\section{9 a 22 de outubro de 2014 \\ Florianópolis/SC}

\section{CONCLUSÕES}

No presente trabalho, foi proposta uma promissora estratégia na produção de biolubrificantes por hidroesterificação do óleo de macaúba. O processo consistiu em uma etapa sequencial de hidrólise da oleaginosa, utilizando um biocatalisador de baixo custo e altamente ativo a partir de sementes dormentes de mamona para produzir concentrado de AGL, seguido pela esterificação enzimática dos AGL com hexanol em meio de heptano mediada por lipase microbiana imobilizada em suporte hidrofóbico, biodegradável e de baixo custo (PHB). O processo sequencial forneceu $\approx 92 \%$ de conversão em ésteres hexílicos (biolubrificantes) após $2 \mathrm{~h}$ de reação, mostrando ser um processo bastante atrativo para a obtenção de compostos de interesse industrial como biolubrificantes.

\section{AGRADECIMENTOS}

Os autores agradecem à FAPEMIG (Processo APQ-00968-12), CNPq (Processo 475289/20129), CAPES e FINEP pelo suporte financeiro. Os autores também agradecem à FAPEMIG e ao CNPq pela concessão de bolsas à J. J. Bassi e A. P. P. Bressani, respectivamente.

\section{REFERENCIAS}

AKERMAN, C.O.; GABER, Y.; GHANI, N.A.; LAMSA, M.; HATTI-KAUL, R. Clean synthesis of biolubricants for low temperature applications using heterogeneous catalysts. J. Mol. Catal. B: Enzym., v. 72, p. 263-269, 2011.

CHOWDHURY, A.; MITRA, D.; BISWAS, D. Biolubricant synthesis from waste cooking oil via enzymatic hydrolysis followed by chemical esterification. J. Chem. Technol. Biotechnol., v. 88, p. 139-144, 2013.

CHOWDHURY, A.; CHAKRABORTY, R.; MITRA, D.; BISWAS D. Optimization of the production parameters of octyl ester biolubricant using Taguchi's design method and physicochemical characterization of the product. Ind. Crops Prod., v. 52, p. 783-789, 2014.

GRYGLEWICZ, S.; PIECHOCKI, W.; GRYGLEWICZ, G. Preparation of polyol esters based on vegetable and animal fats. Bioresour. Technol., v. 87, p. 35-39, 2003.

IHS Incorporation. http://press.ihs.com/press-release/ihs-chemical/growing-lubricants-market-brazildriven-significant-automotive-demand-acc - Acessado em 19 de abril de 2014.

MIRANDA, J.S.; SILVA, N.C.A.; BASSI, J.J.; CORRADINI, M.C.C.; LAGE, F.A.P.; HIRATA, D.B.; MENDES, A.A. Immobilization of Thermomyces lanuginosus lipase on mesoporous polyhydroxybutyrate particles and application in alkyl esters synthesis: Isotherm, thermodynamic and mass transfer studies. Chem. Eng. J. (2014). Trabalho submetido.

SANTOS, K.C.; CASSIMIRO, D.M.J.; AVELAR, M.H.M.; HIRATA, D.B.; CASTRO, H.F.; FERNÁNDEZ-LAFUENTE, R.; MENDES, A.A. Characterization of the catalytic properties of lipases from plant seedsfor the production of concentrated fatty acids from different vegetable oils. Ind. Crops Prod., v. 49, p. 462-470, 2013. 